Résumés des conférences et travaux

\title{
Études ottomanes
}

\author{
Nicolas Vatin
}

\section{OpenEdition \\ Journals}

Édition électronique

URL : https://journals.openedition.org/ashp/973

DOI : 10.4000/ashp.973

ISSN : 1969-6310

Éditeur

Publications de l'École Pratique des Hautes Études

Édition imprimée

Date de publication : 2 février 2011

Pagination : $42-45$

ISSN : 0766-0677

Référence électronique

Nicolas Vatin, «Études ottomanes », Annuaire de l'École pratique des hautes études (EPHE), Section des sciences historiques et philologiques [En ligne], 141 | 2011, mis en ligne le 23 février 2011, consulté le 06 juillet 2021. URL : http://journals.openedition.org/ashp/973 ; DOI : https://doi.org/10.4000/ashp.973 


\title{
ÉTUDES OTTOMANES
}

\author{
Directeur d'études : M. Nicolas VATiN
}

Programme de l'année 2008-2009: Traduction et commentaire des Ġavazât de Hayrü-d-dîn Paşa.

Le séminaire de l'année 2008-2009 a principalement été consacré aux Gazavât-ı Hayrü-d-dîn Paşa (Geste de $\underline{H}$ ayrü-d-dîn Paşa), biographie des célèbres frères Barberousse : $\underline{H} 1$ żır (alias Hayrü-d-dîn) et son aîné Oruç, corsaires turcs qui se taillèrent un domaine au Maghreb au début du XVI siècle avant d'en faire hommage au sultan ottoman. Oruç mourut en 1517 près de Tlemcen; Hayrü-d-din Paşa quant à lui était à sa mort, le 4 juillet 1546, beylerbey des îles, patron de la flotte impériale ${ }^{1}$.

Les Gazavât sont l'œuvre d'un certain Seyyîd Murâd², écrivain professionnel qui dut mettre la main à la seconde version du Kitâb-ı bahriye de Pîrî Re'îs (et en composer l'introduction versifiée) et fut attaché en 1534 à Hayrü-d-dîn quand Soliman appela celui-ci auprès de lui, apparemment chargé de rédiger la geste du pacha. On lui doit un

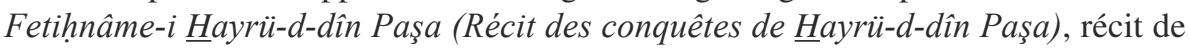
l'expédition des Pouilles (1537-1538) achevée par la bataille de Preveza (inséré par la suite dans la version versifiée des $\dot{G} a z a v a ̂ t$ ) et en 1539 un poème intitulé La conquête de Castelnuovo (Feth-i kal 'e-i Nova). Puis vinrent les Gazavât-ı Hayrü-d-dîn Paşa, qui commencent par l'installation à Mytilène du père des Barberousse et s'achèvent brusquement avec le siège d'Alger par Charles Quint en 1541. Seyyîd Murâd semble avoir alors interrompu son travail pour suivre, au service du grand vizir Rüstem Paşa, les campagnes de Hongrie, écrivant la Ta'rîh-i feth-i Siklôş Usturĝun ve Ustûn-l Belgrâd (Histoire de la conquête de Siklós, Esztergom et Székesfehervar). Mais il

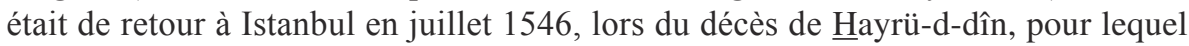
il rédigea huit chronogrammes. Quelques semaines plus tard, en septembre 1546, il acheva la suite des $\dot{G} a z a v a ̂ t$ en prose (de l'arrivée de Hayrü-d-dîn en France en 1543 à sa mort en 1546), texte dont l'unique manuscrit est conservé à la Bibliothèque nationale de France ${ }^{3}$. On perd ensuite sa trace.

Les Gazavât-ı Hayrü-d-dîn Paşa constituent une source bien connue : Hammer en fit usage, soit directement, soit à travers Kâtib Çelebi qui en avait fait sa source. Quant aux historiens occidentaux, notamment les spécialistes du Maghreb, ils avaient à leur disposition diverses traductions. Il en existait une en espagnol, manuscrite, de 1578, faite par un esclave ottoman connaissant l'espagnol, qui fut retraduite et publiée en

1. Le terme kapûdân paşa n'apparut que plus tard.

2. Sur l'attribution des Ġazavât-ı Hayrü-d-dîn Paşa à Seyyîd Murâd et la personne de celui-ci, cf. les p. 3-7 et 10-25 de 1'introduction de Aldo Gallotta, « Il "Ġazavât-1 Hayreddîn Paša" di Seyyid Murâd », Studi Magrebini, XIII (1981).

3. Mauscrits orientaux, Supplément turc 1186. 
italien dans les années $1880^{1}$, et qui a depuis été publiée dans sa version espagnole. D'autre part, Venture de Paradis avait trouvé, lors d'un séjour à Alger en 1788-1789, une version arabe, qu'il traduisit en français : traduction publiée en 1837 par Sang et Denis $^{2}$, qui pensaient qu'il s'agissait d'un texte arabe original du $\mathrm{XVI}^{\mathrm{e}}$ siècle dû à un proche de Barberousse. C'est A. Berbrugger qui identifia le manuscrit et signala qu'il était une traduction du turc ${ }^{3}$. La version française publiée par Sang et Denis, généralement considérée comme médiocre et qui n'était que la traduction d'une traduction, servit de source aux historiens. Il m'a donc paru qu'il ne serait pas inutile de préparer, à l'occasion de mon séminaire, une traduction française à partir de l'original ottoman.

Ce travail, qui sera de longue haleine, est rendu possible par les travaux d'Aldo Gallotta, qui a étudié la tradition manuscrite et publié le texte en prose sous la forme d'un fac-similé, accompagné d'un apparat critique complet du manuscrit qui lui paraissait le meilleur ${ }^{4}$ : celui conservé à l'Escurial à Madrid, qui est antérieur à 1578, date de sa traduction espagnole (ms. $\left.\mathrm{E}^{5}\right)$. A. Gallotta a montré qu'il devait procéder d'un même manuscrit (inconnu) que celui conservé au Vatican (ms. $\left.\mathrm{V}^{6}\right)$, lui-même antérieur à 1566, sinon même à 1543 (date du décès du prince Mehmed, auquel il pourrait avoir appartenu). Dans un premier article de 1970, A. Gallotta était parvenu à une autre conclusion, considérant que le ms. V appartenait à la même famille que les $\mathrm{mss} \mathrm{B}^{7}$, $\mathrm{J}^{8}$ et $\mathrm{I}^{9}$. De fait, la version du ms. V paraît souvent plus proche de l'autre famille de manuscrits que de celle du ms. E. Mais il est vrai que dans son article de 1970, A. Gallotta ne se fondait que sur les premiers folios et qu'il a travaillé sur l'ensemble du texte dans l'introduction à sa publication. On pourra donc le suivre quand il affirme la parenté de V et E. En revanche, on peut s'interroger sur le choix du ms. E pour base de son édition : en effet, le ms. V - le plus ancien, et qui donne fréquemment un texte plus satisfaisant - adopte souvent la même version que les manuscrits du groupe B-J-I. En pareil cas, il paraît généralement préférable d'opter pour la version V. En effet, si celle-ci est de la même famille que le manuscrit de l'Escurial, il faut conclure que quand elle donne la même leçon que les manuscrits de l'autre famille, cette leçon émane d'un manuscrit plus ancien dont procèdent tous les manuscrits connus. Précisons du reste que ces divergences n'ont que rarement des conséquences pour l'interprétation historique du texte ${ }^{10}$.

Ainsi que nous l'apprend Seyyîd Murâd lui-même $\left(2 v^{\circ}-3 r^{\circ}\right)$, c'est Soliman le Magnifique qui commanda sa biographie au pacha :

1. E. Palaez, La vita e la storia di Ariadeno Barbarossa, Palerme 1887; $1^{\text {re }}$ publication dans Archivio Storico Italiano, 1880-1887; rééd. en 1993.

2. R. Sang et F. Denis, Fondation de la Régence d'Alger. Histoire des Barberousse, 2 vol., Paris, 1837.

3. A. Berbrugger, Les époques militaires de la Grande Kabylie, Alger, 1857, p. 309-310.

4. A. Gallotta, « Il "Ġazavât-1 Hayreddîn Paša" di Seyyid Murâd ", art. cit. Cet auteur avait précédemment publié un important article, repris, corrigé et complété dans l'introduction de son édition : « Le Ġazavât di Hayreddîn Barbarossa », Studi Magrebini, III (1970), p. 79-160.

5. Madrid, Escurial, $n^{\circ} 1663$.

6. Biblioteca Vaticana, Barberiniano Orientale $\mathrm{n}^{\circ} 127$.

7. Berlin, Staatsbibliotek, or. Quart 1751.

8. Istanbul, Üniversite Kütüphanesi, $n^{\circ} 94$.

9. Istanbul, Üniversite Kütüphanesi, n 2459.

10. Plusieurs éditions turques en caractères latins ont paru, mais elles ne sont pas fondées sur les meilleurs manuscrits. 
Tout cela et toutes les $\dot{g} a z \hat{a}$ et tous les combats que vous avez menés jusqu'à ce jour sur terre et sur mer, petits et grands, tu vas en faire une composition sans lacune allant des racines aux branches, des débuts à la fin. Tu en feras deux livres détaillés, en vers et en prose, que tu enverras à mon seuil de félicité, en sorte qu'on l'ajoute à sa place, sans ajout ni lacune, aux histoires qui narrent ce qui s'est passé sous mon règne $\left(2 \mathrm{v}^{\circ}\right)$.

Il s'agissait donc de contribuer, par la rédaction de la geste des Barberousse, à l'histoire et à la célébration du règne. On sait du reste que ce sultan suscita une littérature historique (hagiographique selon Ahmet Uğur) sur son père Selîm ${ }^{1}$, et fut à l'origine de la première tentative de création d'une fonction d'historiographe officiel (şehnâmeci) ${ }^{2}$. Cette littérature historique pouvait être de haute tenue littéraire, mettant par là même en valeur la gloire sans pareille de la dynastie. Les premiers şehnâmeci rédigeaient du reste en persan, la langue littéraire par excellence pour un Ottoman. Murâdî pouvait lui aussi donner une forme littéraire à ses travaux (ou ceux de Pîrî Re'îs) en les versifiant.

Mais le récit historique avait un autre usage, de propagande plus immédiatement politique à l'intention de la population. C'est pourquoi la geste des Barberousse appartient au genre traditionnel des gazavât : récits épiques de combats contre les infidèles, rédigés en une langue accessible aux milieux populaires ${ }^{3}$. On est à mi-chemin de la littérature orale, comme le montrent la présence d'interjections et la composition de certains manuscrits en meclis («séances » de lecture). Ici et là, le récit historique prend une forme édifiante ou fantastique, ou tout simplement celle du roman de cape et d'épée. Aussi les Gazavâz-ı Hayrü-d-dîn Paşa connurent-elles un grand succès populaire, dont témoigne le nombre des manuscrits.

Pour autant on aurait tort de n'y voir que « des histoires ». C'est aussi de l'histoire. Seyyîd Murâd prend soin de rappeler qu'il a puisé aux meilleures sources : sa propre expérience, ou les récits du pacha ou de ses compagnons ${ }^{4}$. À l'expérience de l'analyse critique, l'anecdote apparemment la plus fantaisiste peut se révéler fondée ${ }^{5}$. C'est donc une source de première valeur.

C'est particulièrement vrai pour les premières années des frères Barberousse, pour lesquelles les Ǵazavât constituent la seule source fiable ${ }^{6}$. C'est précisément à cette période que sont consacrés les dix huit premiers folios, qui ont été lus, traduits et commentés cette année. Nous y apprenons qu'Oruç, Hayrü-d-dîn et leurs frères étaient les

1. Cf. Ahmed Uğur, The Reign of Sultan Selîm I in the Light of the Selîm-nâme Literature, Berlin, 1985.

2. C. Woodhead, «An experiment in official historiography? The post of şehnâmeci in the Ottoman Empire », Wiener Zeitschrift für die Kunde des Morgenlandes, 75 (1983), p. 157-182.

3. «En langue turque pour être plus accessible aux auditeurs» $\left(3 \mathrm{v}^{\circ}\right)$.

4. «J'ai donc écrit [ce livre] en me fondant pour certains chapitres sur les propos bénis du [pacha] en personne, pour d'autres sur ses frères combattants de la foi qui l'avaient accompagné dans ses gazâ, pour d'autres enfin sur ce que moi, cet humble individu, j'avais moi-même vu et appris alors que je l'accompagnais dans ses campagnes et $\dot{g} a z \hat{a}$ sur terre et sur mer, en sorte qu'il n'y ait pas trace de doute sur sa véracité $»\left(3 \mathrm{v}^{\circ}\right)$.

5. Sur ce point, cf. N. Vatin, «À propos de la captivité à Rhodes d'Oruç Re'îs dans les Ġazavât-ı Hayrüd-dîn Paşa », dans U. Marazzi (éd.), Turcica et Islamica. Studi in memoria di Aldo Gallotta, Naples, 2003, vol. II, p. 995-1011.

6. Cf. Svat Soucek, « The rise of the Barbarossas in North Africa », Archivum Ottomanicum, III (1971), p. 238-250 ; Id., « Remarks on some Western and Turkish Sources dealing with the Barbarossa brothers », Güney-doğu Avrupa Araştırmaları Dergisi, I (1972), p. 63-76. 
fils d'un soldat ottoman ${ }^{1}$ originaire de Yenice Vardar (Gianitsa) installé par Meḥmed II à Mytilène et d'une fille grecque insulaire de bonne famille. De ce fait bilingues, les frères étaient partout à leur place en Méditerranée orientale, où ils commencèrent leur carrière comme marchands et capitaines de navires marchands, ce qui donne à penser que leur famille maternelle était assez riche. Leur sort bascule quand Oruç est attaqué et capturé par des corsaires rhodiens. Averti, son frère $\underline{\text { H}} 1$ ịır se hâte d'aller à Bodrum (place des chevaliers de Rhodes) pour négocier son rachat, mais Oruç réussit une évasion spectaculaire et entame bientôt une nouvelle carrière. Le récit de sa captivité et de son évasion $\left(6 \mathrm{v}^{\circ}-16 \mathrm{v}^{\circ}\right)$ sont caractéristiques de la nature du texte. D’une part on $\mathrm{y}$ trouve nombre de détails exacts et des indications assez précises pour permettre non seulement de dater l'épisode (fin de l'été 1508-début du printemps 1509), mais encore d'identifier les deux chevaliers de l'Ordre de Saint-Jean propriétaires du héros, que Seyyîd Murâd ne nomme pourtant pas : le maréchal Bertrand Corault et le turcopelier Robert Daniel ${ }^{2}$. D'autre part, on retrouve les poncifs du récit de captivité chez l'infidèle (discussion sur la foi, refus de se convertir au christianisme) et le récit de l'évasion est à la fois concret - Oruç, qui a préalablement scié ses fers, profite d'une tempête près des côtes anatoliennes et de l'absence momentanée du canot de la galère sur laquelle il rame - et merveilleux : il ne doit sa liberté qu'à sa piété, car Mahomet, dont il a demandé le secours par ses prières, est venu en personne le libérer. C'est en tout cas ce qu'il fait savoir aux chevaliers de Rhodes...

On voit que dans ces premières pages Hayrü-d-dîn répondait, en dictant ses souvenirs à Seyyîd Murâd, à une question que lui avait posée Soliman :

Or donc, comment êtes-vous apparus toi et ton frère? Quelles furent la raison et la cause de votre apparition et votre essor? Quel genre de personnes êtes-vous : êtes-vous issus de la catégorie des $k u l$, ou étiez-vous extérieurs [au service du sultan]? $\left(2 \mathrm{v}^{\circ}\right)$.

Formule qui donne à entendre qu'on ne savait pas grand chose, en 1541, de ces aventuriers à qui l'Empire devait ses territoires maghrébins. On peut même se demander si leur réputation de corsaires ne paraissait pas un peu compromettante pour un pacha du sultan. À cela, les Ġazavât répondaient, tant aux contemporains qu'à la postérité, que les frères Barberousse étaient musulmans de naissance, fils d'un serviteur de la dynastie que Hayrü-d-dîn servait maintenant au plus haut niveau et d'une fille de bonne famille; que d'ailleurs, loin d'avoir été des corsaires (sinon même des pirates), ils étaient par vocation d'honnêtes marchands. Il n'y a pas de raison de douter de l'activité de commerce d'Oruç et Hayrü-d-dîn à leurs débuts - tout en rappelant qu'elle n'excluait pas nécessairement toute activité corsaire -, mais on peut néanmoins soupçonner qu'il y a une volonté de propagande dans l'insistance sur ce point de Seyyîd Murâd : en démontrant l'honorabilité du pacha, il coupait court à des bruits qui auraient pu ternir la gloire du règne de Soliman ${ }^{3}$.

1. Le statut de ce Ya'kûb n'est pas entièrement clair : il semble bien être un $k u l$ et un homme de garnison, donc un janissaire, mais il est également présenté comme un « sipahi, lui-même fils de sipahi » $\left(5 \mathrm{v}^{\circ}\right)$.

2. Cf. N. Vatin, art. cit.

3. J'ai sur cette question rédigé un article à paraître à Istanbul dans un volume à la mémoire de Stéphane Yerasimos, sous le titre " "Comment êtes-vous apparus, toi et ton frère ?" Note sur les origines des frères Barberousse ». 